\title{
IEF2C: A novel Al-powered framework for suspected COVID-19 patient detection and contact tracing in smart cities
}

\section{Mohammad Azazur Rahman}

Dhaka University of Engineering \& Technology (DUET), Gazipur

Md. Tareq Mahmud ( $\nabla$ tareq.iict@duet.ac.bd)

Dhaka University of Engineering \& Technology (DUET), Gazipur https://orcid.org/0000-0001-6549701X

\section{Md. Obaidur Rahman}

Dhaka University of Engineering \& Technology (DUET), Gazipur

\section{Research Article}

Keywords: Novel coronavirus, COVID-19, Pandemic, Smart city, Contact tracing, Framework

Posted Date: October 6th, 2020

DOl: https://doi.org/10.21203/rs.3.rs-87143/v1

License: (a) (1) This work is licensed under a Creative Commons Attribution 4.0 International License. Read Full License 


\title{
IEF2C: A novel AI-powered framework for suspected COVID-19 patient detection and contact tracing in smart cities
}

\author{
Mohammad Azazur Rahman' ${ }^{1}$ Md. Tareq Mahmud1, 2 (iD . Md. Obaidur Rahman', 2
}

Received: date / Accepted: date

\begin{abstract}
Novel coronavirus disease 2019 (COVID-19) has found as a climacteric pandemic that spreads from humanto-human through contiguity. It is not possible to control the virus properly because its vaccine has not been discovered yet. As of now, various contact tracing approaches are being taken to contain the spread of the virus by breaking its transmission chain. Recently, few technologies are being exploited for contact tracing such as geological location, Bluetooth and google maps. Governments of some countries have already launched contact tracing applications to identify the close contact people of COVID-19 positive patients. However, these methods may not be effective without a huge amount of testing facility since the tracing action starts when a COVID-19 testing result is found positive. In this paper, we have proposed a novel AI-powered data management framework for smart city named IoT-to-Edge-toFog-to-cloudlet-to-Cloud (IEF2C) data management framework which will be exploited to identify the COVID-19 suspected patients in smart cities and also be used to inform the surrounding people about the suspected patient. The proposed framework is isolated into five layers which have different tasks such as data collection, processing, authentication and storage. The framework will bring all the citizens under a common platform as well as will ensure the user and device mobility. It will mitigate the information gap by
\end{abstract}

Mohammad Azazur Rahman · Md. Tareq Mahmud (凶) $\cdot$ Md. Obaidur Rahman

${ }^{1}$ Institute of Information \& communication Technology (IICT), Dhaka University of Engineering \& Technology (DUET), Gazipur, Bangladesh

${ }^{2}$ Department of computer Science \& Engineering, Dhaka University of Engineering \& Technology (DUET), Gazipur, Bangladesh

E-mail: ezaz.ctgbd3@gmail.com, tareq.iict@ duet.ac.bd (凶), orahman@duet.ac.bd helping concerned authorities and users to take proper steps in such a pandemic era.

Keywords Novel coronavirus · COVID-19 - Pandemic · Smart city $\cdot$ Contact tracing $\cdot$ Framework

\section{Introduction}

Novel coronavirus disease 2019 (COVID-19) is a pandemic that brings about intensive illness and the demise of the human. COVID-19 is caused by the SARS-Cov-2(severe acute respiratory syndrome coronavirus 2 ) virus. The consequence of COVID-19 includes intensive panic and economic transiency all over the world [1,2]. In December 2019, the outbreak of the COVID-19 commenced in Wuhan, the most densely populated city in Central China and the capital of Hubei Province of the People's Republic of China. The novel coronavirus 2019 (COVID-19) has spread incredibly in 210 countries all over the world and officially announced as a global pandemic by the World Health Organization (WHO) on April 25, 2020 [3,4].

COVID-19 is extremely infectious for the median duration of 4 days during the process of incubating and gives rise to Pneumonia with usual clinical symptoms of fever, fatigue and dry cough. Few additional symptoms may arise such as dyspnea, diarrhea, headache, myalgia, mucus or phlegm, hemoptysis and nausea $[5,6,7]$. A huge number of people contaminated with COVID-19 will encountered respiratory sickness and recoup without requiring exceptional treatment. However, the elderly community and the people with basic clinical issues such as chronic respiratory infection, diabetes, cardiovascular disease and cancer, have the probability to experience of severe illness $[8,9,10]$. The virus spreads from human-to-human through an infected person's contact transmission with droplets of cough or sneezes [11]. 


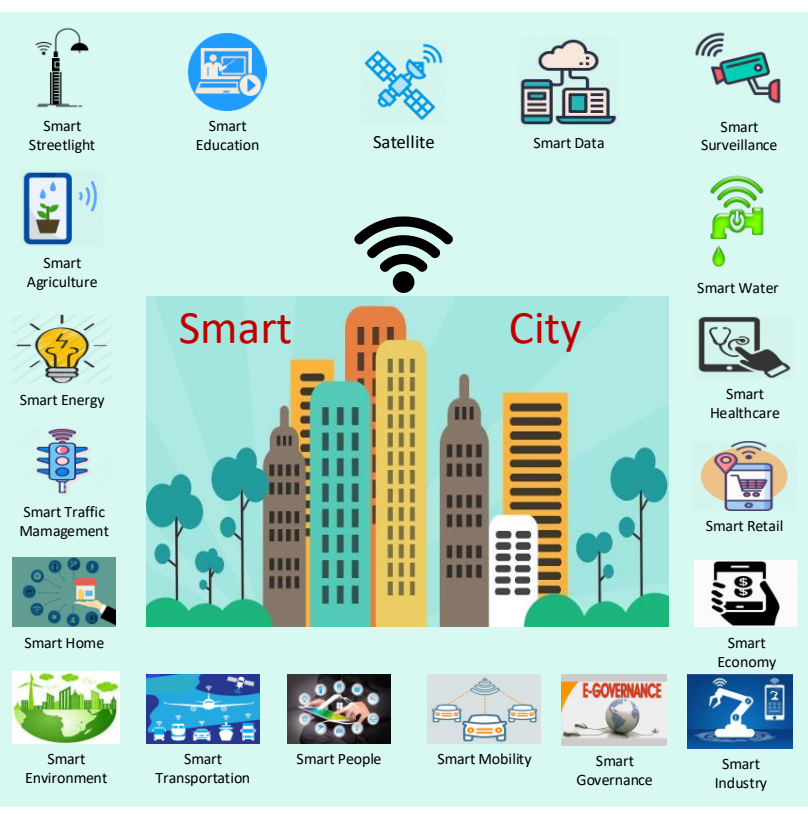

Fig. 1 Smart city architecture overview.

Researchers are trying to invent an effective vaccine for COVID-19. However, it is not a simple process and therefore, it is certainly taking a longer time. To keep safe from the COVID-19, people should maintain some rules such as avoiding physical touch, social distance, isolation, wearing protective equipment until the vaccine comes [12].

Nowadays, a smart city is an emerging technological concept. It will connect all the components of a city, such as- office, hospital, hotel, smart grid, home and other necessary functional infrastructures via the emerging technology Internet of Things (IoT) (as demonstrated in Fig. 1). Smart city concept will be able to ensure public services efficiently in real-time. Researches expect that about $70 \%$ of people in the world will live in the city within 2050 [13]. So, it is an alarming policy making issue for the authorities because the cities will have to manage a vast population and thereby the possible smart city concept is no longer a dream. In a smart city, all the smart devices will integrate into a central system [14]. The service providers in the city will get the necessary access of respective information from the system and will act accordingly [15].

A smart city will explore the governance services vastly to the mass people. City authority will be able to provide service efficiently so that development in all places of a city will be easy. Thus, surely it will introduce various techniques to the citizen for daily living purposes. Smart city diagram can use the blockchain technology for identifying the attributes of the citizens and personal characteristics [16]. The smart traffic management system will be developed by smart city concepts such as smart parking, integrated model transport, crowdsourcing and so. People can save time and accidental damage. Moreover, the smart city will be able to monitor environmental conditions continuously, so it will be easy to take proper action in terms of keeping the environment stable. By using video surveillance, the smart city will ensure surveillance system a secure situation in the city, so city dwellers will feel secure and safe.

Now a days, Artificial Intelligence (AI) is playing a vital role in our daily life. AI has already been implemented in IoT devices but it is limited in-home condition. In future, it expected to be applied broadly, especially to operate the IoT based devices from Cloud [15,17]. Note that, appliances of our daily life will also be connected with the Cloud via IoT [17].

Health management of the community of a city or country will be directly aligned with smart city technology in the future. Hospital authorities will get the health information of a citizen by taking the user's permission [18]. The doctor will easily be able to understand the proper scenario of a patient. Moreover, health management will take the proper steps in an emergency situation. As a smart city will be mapped in an integrated network, so the hospitals, clinics, diagnostics and other health-related organizations will come into a common platform. The health service can be ensured within a very short time.

Considering the global pandemic effect of COVID-19, many research has been going on various issue. Among them contact tracing is one of the major concern right now to mitigate the vast effect of COVID-19. The World Health Organization (WHO), governments and health organizations cannot take proper steps due to the lack of proper information about infected people. So, contact tracing, isolating the suspected and infected people are the most effective ways to mitigate the spread of the pandemic. Contact tracing is a process in order to mitigate the spread of a virus. It is also an algorithm of identifying, measuring and managing people who have been exposed [12]. The method helps to break the virus transmission chain. In case of COVID-19, researchers are applying different types of technology for contact tracing such as geological location [18], Bluetooth [19], google maps [20]. Generally, all of these contact tracing strategies are found effective when a COVID-19 patient's testing result become positive; therefore, these methods may not be efficacious without an enormous amount of testing facility. In most countries, the COVID-19 testing facilities are not sufficient with respect to demand. As a result, a huge number of infected people are out of identification. The contact tracing method can be fruitful in those countries/places where most people can not avail the testing facility of COVID-19 identification. So, the identification of a suspected person is the first challenge to control the virus outbreak.

In this paper, we have proposed an AI-powered data management framework named IoT-to-Edge-to-Fog-to-cloudletto-Cloud (IEF2C) which will perform more efficiently and process the data of smart city intelligently. Our data man- 
agement framework of smart cities can play a vital role to make the contact tracing more effective. It will alert people about a suspected person even if the person is not tested for COVID-19 identification. The framework uses the symptomatic health data of the citizens to identify the COVID-19 suspected. This framework will be able to connect multiple smart cities in a common framework. So, the mobility of user devices and data will be possible. Five layers are used in this framework to integrate all IoT-enabled sensors and devices of smart cities. IoT layer is the lowest layer of the proposed framework and consists of all the IoT/sensor devices of a smart city. The Edge layer has an important role in the framework, where it connects the IoT devices with the functional storage layers and will be able to control the devices by its own data management system on emergency. Moreover, the Fog layer of the proposed data management framework has more functionalities. AI implementation is one of them which will help to reduce the data processing load of the Cloud layer as well as predict whether a citizen is COVID-19 suspected or not. Finally, cloudlet layer is responsible for trace and maintain the user and device mobility.

The remaining part of this paper is organized as follows: Section 2 presents the related works on data management framework of smart cities and COVID-19 contact tracing. Features of data management frameworks in smart cities are discussed in Section 3. In Section 4, our proposed data management framework is presented. The data generated at each of the layers of proposed framework are explained in Section 5. Then, the proposed data management framework is exploited for COVID-19 contact tracing in Section 6. Finally, the paper is concluded in Section 7.

\section{Literature Review}

A smart city provides services and resources to its citizen. The services are classified in some branches such as living, governance, economy, environment, education, healthcare, safety, security $[21,22,23]$. Those services can be ensured by using resources like brainware, hardware, and software [21]. To ensure the services by using the resources, a bunch of technological aspects should be considered with respect to quality features. Cloud computing, IoT, home appliance integrity, data security, wireless sensor network, data mobility will be the technological aspects in the smart city model, and the quality features define as business architecture, data sources, data management, application, operation system, user integration [24].

Cloud computing is one of the preferable data management technologies for developing smart cities [14]. To decrease the data traffic load and latency, other computing methods can be integrated with Cloud computing [14,17]. Cloud is large storage where a huge amount of data can be stored
[14]. It is also exploited for machine learning (ML), data analytics, visualization, and other operations [17]. However, the bulk amount of data of millions of IoT devices in the smart city will make a huge amount of data traffic. To address this issue, the data management of the smart city is divided into some layers [15]. Every layer will perform a different function and maintain a chain of data flow [25]. Moreover, the layers carry different types of data, such as random data, historical data, KPI data, and research data [14].

The COSA-DLC model [26] for smart cities has proposed an data management architecture that combined the Fog and Cloud computing. The proposed data management architecture ensures security and quick data accessibility. Later, the authors of the COSA-DLC model have proposed a new smart city comprehensive data life cycle (SCC-DLC) [27]. It is more efficient than the COSA-DLC model. The Edge data sources are also connected with Fog devices as the COSA-DLC model [26]. However, the significant difference of this research is that the Fog devices are placed under a Fog area and the Fog areas are connected with Fog layer 2. Fog leader is a new concept of this research and it acts as a bridge between IoT devices and Cloud storage. The 6V (volume, variety, variability, veracity and value) challenges have been introduced in [28] and Zero-Emission Neighborhoods architecture has proposed for smart home data management. IoT hub is a new component in this architecture and other elements are the same as previous research of [23] and [27]. The final research of the series replaces IoT hub by the cloudlet layer [14], where a hybrid data management architecture combined with smart city scenario and ZEN center scenario are demonstrated. The architecture helps to reduce data traffic and latency of smart city data management.

Health management is a significant part of smart cities. All the health information of citizens stored in Cloud storage and health authority will be able to establish a synchronized proper management for providing health services. Thus, the patients get the surety of their health information security. However, Cloud-based healthcare has some limitations [29]. As health service is an emergency service and it contains a huge volume of information. Cloud computing has high latency as well as heavy data load problems. So, Cloud computing has the possibility to deliver health data in a delayed manner. But real-time data processing is the most important demand for healthcare services. As a solution, Fog computing can be an alternative for ensuring real-time data management [30]. The Fog computing layer will be a hub between sensors and Cloud storage. Moreover, it reduces complexity and increases reliability.

eHealth system of the smart city faces some problems such as regulations, interoperability, device-network-human interfaces, security, data management, scalability and privacy. A multi-layer architecture including device layer, Fog layer and Cloud layer is proposed in [31]. It is able to make 
reliable data communication from devices to data storage. The article in [32] has proposed a method of data collection from sensors. Here, the collection of data from sensors can provide proper information about the health condition of the users.

The Edge components have an important role in eHealth systems of smart cities. It acts as a gateway between sensors and Cloud networks. A smart eHealth gateway termed UT-GATE is introduced in [33]. The gateway offers several services such as embedded data mining, real-time data processing, local storage. The gateway provides early warning monitoring. The IoT sensors have the opportunity to connect with different communication protocols such as Ethernet, Bluetooth and ZigBee [34]. However, the gateway should have the flexibility to adapt to different types of protocols with different environments simultaneously. A recent work of [35] has proposed a Fog assisted computing architecture. The Fog layer acts as a gateway and it performs the system managing, planning, and executing. The Cloud layer is exploited to analyze health data. Most of the smart healthcare-related researches are based on home condition. A Fog-Cloud architecture based efficient healthcare system for office environment has proposed in [36].

COVID-19 is one of the crying threat to the health sector now. Almost all the health management of governments are collapsed in the pandemic. As it spreads from person to person and vaccine has not been invented finally yet. So, physical distance and isolation are the only ways to protect. Contact tracing is a prominent method to decrease the flow of spreading viruses. It can be easily exploited by using a mobile phone. In [18], the researchers have proposed a Mobile Cellular Networks-based contact tracing method. The method uses the geolocation data of mobile phone users for contact tracing. Singapore Ministry of Health has already launched a contact tracing app called "Trace Together" [19]. Each of the users have to have their own smartphone for using that solution is found as the key limitation of this concept. Another Bluetooth-based contact tracing app is jointly released by the government of Germany, France and Italy [37]. In a recent article [38], privacy preventing Mobile and Fog Computing framework is implemented. It is also based on Bluetooth technology and posses the limitations of Bluetooth technology. Google Maps timeline (GMTL) is also exploited for getting spatial epidemiologic timelines in [20]. The method compares the locations, routes and means of transport.

The existing contact tracing apps use various communication technologies such as cellular networks, GPS, Bluetooth, WiFi. An epidemic model has been designed to evaluate the efficient contact tracing app and those communication technologies have been applied for simulation purposes [39]. The contact tracing app has some attributes such as data management, system architecture, privacy, proximity, security estimation and vulnerability [40]. To make the contact tracing system successful, COVID-19 testing should be increased. Now there are few methods of testing COVID19 such as Polymer Chain Reaction (PCR), Rapid Antibody Test [41]. However, those testing methods are time-dependent and costly. Clinical Chest CT image [42] and X-ray images [43] are also used for identifying COVID-19 patients. Table 1 reviews the articles that provides the layered architecture for data management of different smart city components, such as COVID-19 contact tracing.

\section{Features: Smart City Data Management Framework}

Data management is an essential aspect of developing a smart city because smart city concept can gather some related technologies such as user and data mobility, AI implementation, IoT, and Cloud-based information. A smart city data management framework should have the following features:

Data Mobility: Nowadays, people shift from one geographical location to another or shift home and office components to the new address. However, if the already stored data of a user is transfered to a new destination via the Cloud, it will reduce the hassle of shifting the home instruments. But it is not feasible for many different locations, as because the weather characteristics (i.e., temperature, atmospheric pressure, wind, solar irradiance, humidity, precipitation, and topography) of two different countries are not the same. Hence, it will be difficult to operate the appliances of the new location by using previously stored data and information.

AI Implementation: Artificial intelligence (AI) will play a vital role in future smart city data management. AI coordinates the IoT devices and makes the user's life more comfortable. It will help the devices to make a decision in an emergency situation and also build a bridge to the neighbor to share information among them.

IoT: All the IoT appliances and devices are connected with the Cloud through Internet and create a synchronized platform. So the user will be able to up to date about the workplace, home and all over the city. Moreover, all service departments of the city will be able to provide services efficiently because of proper data management. 


\begin{tabular}{|c|c|c|c|c|c|c|c|c|c|c|c|}
\hline \multirow{3}{*}{$\begin{array}{r}\text { Article } \\
\text { Ines et al. }\end{array}$} & \multirow{3}{*}{$\frac{\text { Ref. }}{[17]}$} & \multirow{3}{*}{$\frac{\text { Year }}{2019}$} & \multirow{2}{*}{ Domain } & \multirow{2}{*}{ Objective } & \multicolumn{5}{|c|}{ Technologies used in Layered Approach } & \multirow{2}{*}{ Advantages } & \multirow{2}{*}{ Limitations } \\
\hline & & & & & IoT & Edge & Fog & cloudlet & Cloud & & \\
\hline & & & Public Building & $\begin{array}{l}\text { To build smart energy efficient } \\
\text { system for public building }\end{array}$ & $\checkmark$ & $\checkmark$ & $x$ & $x$ & $\checkmark$ & $\begin{array}{l}\text { Reduced data transfer } \\
\text { from IoT Edge to Cloud }\end{array}$ & $\begin{array}{l}\text { Latency for AI operation, } \\
\text { processing in Cloud }\end{array}$ \\
\hline Farzad et al. & [15] & 2019 & $\begin{array}{l}\text { Internet of } \\
\text { Things (IoT) }\end{array}$ & $\begin{array}{l}\text { To analyze use of Machine Learn- } \\
\text { ing (ML) in diverse IoT domains }\end{array}$ & $\checkmark$ & $\checkmark$ & $\checkmark$ & $x$ & $\checkmark$ & $\begin{array}{l}\text { Involve intelligence to } \\
\text { the Edge and IoT device }\end{array}$ & $\begin{array}{l}\text { User and device mobility } \\
\text { are not considered }\end{array}$ \\
\hline Arpna et al. & [25] & 2020 & Smart Home & $\begin{array}{l}\text { To attain Edge Intelligence in real } \\
\text { time processing in Smart Home }\end{array}$ & $\checkmark$ & $\checkmark$ & $x$ & $x$ & $\checkmark$ & $\begin{array}{l}\text { Ensures security and pri- } \\
\text { vacy of user data }\end{array}$ & $\begin{array}{l}\text { AI and ML models are } \\
\text { not utilized }\end{array}$ \\
\hline Amir et al. & [26] & 2016 & Smart City & $\begin{array}{l}\text { To develop an efficient data man- } \\
\text { agement framework }\end{array}$ & $x$ & $\checkmark$ & $\checkmark$ & $x$ & $\checkmark$ & $\begin{array}{l}\text { Data privacy of Fog asso- } \\
\text { ciates Cloud processing }\end{array}$ & $\begin{array}{l}\text { User and device mobility } \\
\text { are not considered }\end{array}$ \\
\hline Jiong et al. & [13] & 2014 & Smart City & $\begin{array}{l}\text { To design an IoT-based frame- } \\
\text { work for smart cities. }\end{array}$ & $\checkmark$ & $x$ & $x$ & $x$ & $\checkmark$ & $\begin{array}{l}\text { Ensures quality services } \\
\text { for citizens. }\end{array}$ & $\begin{array}{l}\text { User and device mobility } \\
\text { are not considered. }\end{array}$ \\
\hline Colistra et al. & [32] & 2018 & Smart City & $\begin{array}{l}\text { To design house models for smart } \\
\text { health system }\end{array}$ & $\checkmark$ & $x$ & $x$ & $x$ & $x$ & $\begin{array}{l}\text { Ensures quality services } \\
\text { for city citizens }\end{array}$ & $\begin{array}{l}\text { User and device mobility } \\
\text { are not considered }\end{array}$ \\
\hline Amir et al. & [27] & 2017 & Smart City & $\begin{array}{l}\text { Data management framework for } \\
\text { smart city based on Fog-Cloud }\end{array}$ & $\checkmark$ & $x$ & $\checkmark$ & $x$ & $\checkmark$ & $\begin{array}{l}\text { Ensures real-time data } \\
\text { access and collection }\end{array}$ & $\begin{array}{l}\text { User and device mobility } \\
\text { are not considered }\end{array}$ \\
\hline Amir et al. & [28] & 2018 & Smart City & $\begin{array}{l}\text { To design ZEN data management } \\
\text { architecture for smart city }\end{array}$ & $\checkmark$ & $x$ & $\checkmark$ & $x$ & $\checkmark$ & $\begin{array}{l}\text { Vast storage and process- } \\
\text { ing capacity assured }\end{array}$ & $\begin{array}{l}\text { User and device mobility } \\
\text { are not considered }\end{array}$ \\
\hline Amir et al. & [14] & 2019 & Smart City & $\begin{array}{l}\text { Distributed-to-Centralized Data } \\
\text { Management architecture }\end{array}$ & $\checkmark$ & $x$ & $\checkmark$ & $\checkmark$ & $\checkmark$ & $\begin{array}{l}\text { Centralized \& distributed } \\
\text { data management used }\end{array}$ & $\begin{array}{l}\text { Lack of intelligence to } \\
\text { the edge of the network }\end{array}$ \\
\hline Tuan et al. & [29] & 2015 & $\begin{array}{l}\text { IoT-enabled Health- } \\
\text { care System }\end{array}$ & $\begin{array}{l}\text { To exploit Fog computing at } \\
\text { smart gateways at network edge }\end{array}$ & $\checkmark$ & $\checkmark$ & $\checkmark$ & $x$ & $\checkmark$ & $\begin{array}{l}\text { Faster data processing at } \\
\text { smart gateways }\end{array}$ & $\begin{array}{l}\text { User and device mobility } \\
\text { are not considered }\end{array}$ \\
\hline Humberto et al. & [30] & 2020 & Healthcare System & $\begin{array}{l}\text { Fog-based data management ar- } \\
\text { chitecture challenges identify }\end{array}$ & $\checkmark$ & $x$ & $\checkmark$ & $x$ & $\checkmark$ & $\begin{array}{l}\text { Spots challenges of Fog } \\
\text { computing architectures }\end{array}$ & $\begin{array}{l}\text { Ignored data privacy and } \\
\text { device interoperability }\end{array}$ \\
\hline Bahar et al. & [31] & 2018 & $\begin{array}{l}\text { IoT-based eHealth } \\
\text { Ecosystem }\end{array}$ & $\begin{array}{l}\text { Layer-wise application mapping } \\
\text { in a holistic eHealth ecosystem }\end{array}$ & $\checkmark$ & $x$ & $\checkmark$ & $x$ & $\checkmark$ & $\begin{array}{l}\text { Figured out the chal- } \\
\text { lenges of IoT eHealth }\end{array}$ & $\begin{array}{l}\text { User and device mobility } \\
\text { are not considered }\end{array}$ \\
\hline Amir et al. & [33] & 2017 & $\begin{array}{l}\text { Healthcare IoT } \\
\text { System }\end{array}$ & $\begin{array}{l}\text { Placement of e-Health Gateways } \\
\text { at the edge of the network }\end{array}$ & $\checkmark$ & $x$ & $\checkmark$ & $x$ & $\checkmark$ & $\begin{array}{l}\text { Ensures system intelli- } \\
\text { gence, energy efficiency }\end{array}$ & $\begin{array}{l}\text { User mobility and Edge } \\
\text { layer are not considered }\end{array}$ \\
\hline Imam et al. & [35] & 2017 & $\begin{array}{l}\text { Health Monitoring } \\
\text { System }\end{array}$ & $\begin{array}{l}\text { A Fog-assisted IoT-Health data } \\
\text { computing architecture design }\end{array}$ & $\checkmark$ & $x$ & $\checkmark$ & $x$ & $\checkmark$ & $\begin{array}{l}\text { Provides ML data analyt- } \\
\text { ics \& self-ruling system }\end{array}$ & $\begin{array}{l}\text { User and device mobility } \\
\text { are not considered }\end{array}$ \\
\hline Munish et al. & [36] & 2019 & $\begin{array}{l}\text { Healthcare in Smart } \\
\text { Office }\end{array}$ & $\begin{array}{l}\text { Layered Fog-Cloud architecture } \\
\text { of IoT-based remote healthcare }\end{array}$ & $\checkmark$ & $x$ & $\checkmark$ & $x$ & $\checkmark$ & $\begin{array}{l}\text { Ensures highly efficient } \\
\text { and effective healthcare }\end{array}$ & $\begin{array}{l}\text { User and device mobility } \\
\text { are not considered }\end{array}$ \\
\hline Tanvir et al. & [18] & 2020 & $\begin{array}{l}\text { COVID-19 Contact } \\
\text { Tracing }\end{array}$ & $\begin{array}{l}\text { Mobile phone users' geolocation } \\
\text { based COVID-19 contact tracing }\end{array}$ & $\checkmark$ & $x$ & $x$ & $x$ & $\checkmark$ & $\begin{array}{l}\text { Mobile infected detected } \\
\text { except smartphone apps }\end{array}$ & $\begin{array}{l}\text { Relies on Geolocation of } \\
\text { Cellular Networks only }\end{array}$ \\
\hline Jason et al. & [19] & 2020 & $\begin{array}{l}\text { COVID-19 Contact } \\
\text { Tracing }\end{array}$ & $\begin{array}{l}\text { Bluetooth-based COVID-19 con- } \\
\text { tact tracing system deploy }\end{array}$ & $\checkmark$ & $x$ & $x$ & $x$ & $\checkmark$ & $\begin{array}{l}\text { Ensures data protection } \\
\text { and privacy }\end{array}$ & $\begin{array}{l}\text { Suitable for short-range } \\
\text { radio communication }\end{array}$ \\
\hline Kimmo et al. & [37] & 2020 & $\begin{array}{l}\text { COVID-19 Contact } \\
\text { Tracing }\end{array}$ & $\begin{array}{l}\text { Rendezvous-based COVID-19 } \\
\text { contact tracing method design }\end{array}$ & $\checkmark$ & $x$ & $x$ & $x$ & $\checkmark$ & $\begin{array}{l}\text { Keeps users persistently } \\
\text { unidentified }\end{array}$ & $\begin{array}{l}\text { Suitable for short-range } \\
\text { radio communication }\end{array}$ \\
\hline $\begin{array}{l}\text { Whaiduzzaman } \\
\text { et al. }\end{array}$ & [38] & 2020 & $\begin{array}{l}\text { COVID-19 Contact } \\
\text { Tracing }\end{array}$ & $\begin{array}{l}\text { COVID-19 contact tracing based } \\
\text { on Mobile and Fog Computing }\end{array}$ & $\checkmark$ & $x$ & $\checkmark$ & $x$ & $\checkmark$ & Ensures user data privacy & $\begin{array}{l}\text { User and device mobility } \\
\text { are not considered }\end{array}$ \\
\hline
\end{tabular}


Cloud-Based Information: To gather data from all the IoT devices of a smart city, the Cloud-based information system will provide proper data management. In this paper, we have identified four types of data in different layers. Cache data refers to the random information which will be generated from IoT devices and user commands [14]. Gateway of the Edge layer will also save some predefined instruction as a temporary basis for the emergency period. These instructions are also cache data. Operational data comes from both IoT device and user command to the Fog layer and it will be analyzed to develop AI in the appliance's office, hospital, hotel, smart grid, home and other necessary functional components of the smart city. Authentication data will be used to identify the valid user of these smart city components. Archived data will be collected from the Fog layer and saved as backup for emergencies [14].

\section{Proposed IEF2C Data Management Framework}

The activities of the proposed IEF2C data management framework are distributed among five layers. Each layer has the following distinct responsibilities:

\subsection{IoT Layer}

IoT layer is the initial layer of the proposed data management framework. All of the IoT-enabled sensors, appliances, and devices are included in this layer [17]. This layer is the main source of information where all the IoT devices generate all sorts of raw data (as shown in Fig. 2). The layer unit can be an office, hospital, hotel, smart grid, home or any other functional components of a smart city. The sensors and appliances will provide data to the next layer called the Edge layer and the sensor devices will be connected with a controlling device of the Edge layer using different communication systems and protocols. In addition, IoT layer will be controlled and monitored by the service users and AI systems of Fog layer. The service users will allow for accessing only the IoT devices by taking proper authentication from the cloudlet layer.

\subsection{Edge Layer}

The Edge layer will act as a gateway to the IoT Layer. It will play a vital role in the proposed data management framework. This framework aggregates all the information from three ends and those are IoT layer, Fog layer and user. Every user will connect with a particular Edge unit using an authentic user account. This is how a home, office or functional place will connect with an Edge unit to manage the activities of the IoT layer components.
The Edge layer will be maintained by the functional user (as shown in Fig. 3). As it is a gateway, it will be needed to configure and update periodically. The features of the Edge layer are given below:

1. Predefined Instruction: The Edge layer is responsible for an emergency situation. If the Edge layer disconnects with the Fog layer and user, it will be difficult to provide feedback to operate to the IoT layer [25]. In terms of this condition, the Edge layer will set some predefined instructions into its short memory. So, the IoT layer will be able to run the appliances by getting the predefined instruction feedbacks from the Edge layer in the emergency period. The instructions will be always updated after a certain period every time. The predefined instructions can either be set by the users or can be collected from the Fog layer. It will work on the demand of the user's necessity.

2. Storage Capacity: To execute the predefined operations, the gateway needs to store the related instructions. So, the Edge component should have a storage capacity; even though, it is not like the bulk amount similar to Fog layer or Cloud layer.

3. Data Recalling: In this framework, not only the appliance data will be saved to the Fog layer but also be exploited to implement the AI system on the IoT layer devices. When users will not be available to provide instructions, the user devices will operate itself by AI; where data recalling from primary storage will be needed. This function will be done by the Edge layer.

4. Data Segregation: The layer has two command ends. Those are user and Fog layer. The Edge layer will distribute the data which will come from IoT devices or the Fog end. For this purpose, the Edge layer will segregate the data to the proper end.

5. User Device Authentication: To secure the office, hospital, hotel, smart grid, home and any other functional components of a smart city from cyber-attack, the Edge layer will identify the attacker device. It will allow the command only from the Fog layer as well as from the authentic devices.

\subsection{Fog Layer}

This layer can be termed as the Fog computing layer and all the IoT devices of a city will be connected with the Fog database through Edge gateway. That means the Fog layer will be the core data operational centre of a particular smart city. All the data of IoT layer will come to this layer and the layer will analyze the data by using machine learning algorithms or techniques such as k- Nearest Neighbors (kNN), Artificial Neural Networks (ANN), Deep Neural Networks (DNN), Convolutional Neural Networks (CNN) and Recur- 


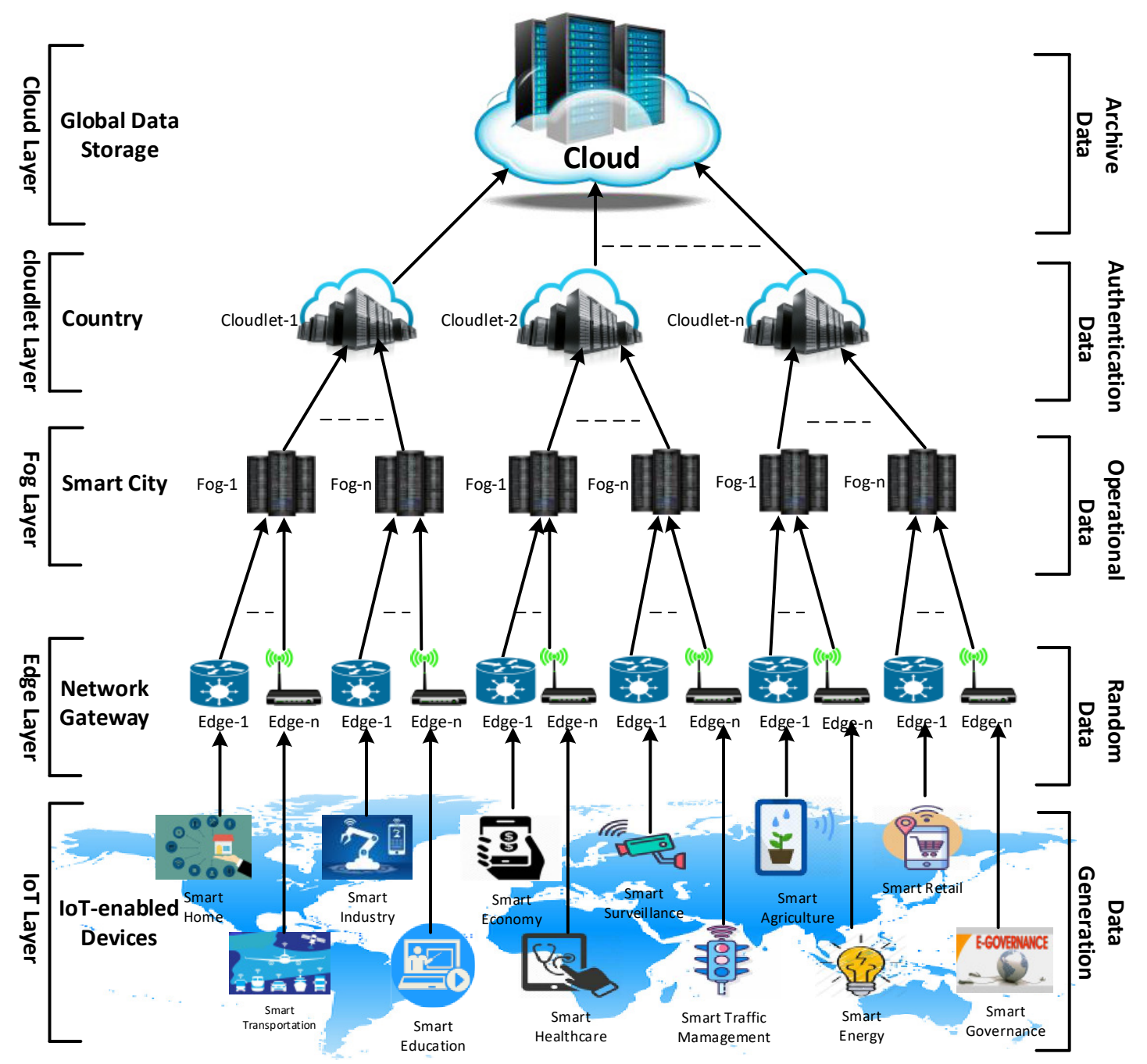

Fig. 2 IEF2C data management framework of a smart city scenario.

rent Neural Networks (RNN) etc. [15]. It will collect the device information and user commands to learn user behavior and activities. So, after a certain period, the AI system of the Fog layer will able to provide proper instructions to the devices of the IoT layer without user's command. In order to analyze, the Fog layer will use random data from the IoT layer and previous data from the cloudlet layer for analysis. It will ensure data security because it will not allow accessing the data in the Cloud or cloudlet layer directly. Moreover, the office, hospital, hotel, smart grid, home and any other functional components of a smart city will connect with the layer, so it will build an interconnection. As a result, it is possible to share some common services among the functional components of a smart city such as powersharing, security alert, social connectivity.

This layer will be accessed by the functional users only who can also access the Edge layer (as demonstrated in Fig. 3 ). The users will monitor the layer's storage security, maintenance and system upgradation.

\section{4 cloudlet Layer}

Mobile Cloud Computing (MCC) method will be applied in the cloudlet layer. A cloudlet unit connects all the Fog unit of a country so that it can act as a data hub of a country. This layer is also exploited to decrease data traffic and latency in the Cloud [26].

The cloudlet layer will play a role as data storage of a country and it will store the data from its subordinate Fog units. It will also act as a hub of data supply from the Fog to the Cloud layer and store all the previous data of the users as well as supply the data to the Fog layers on demand. As a hub, it will perform some functional operations such as data filtering, aggregation, compression and so on. The operations are needed to reduce the data load of the Cloud layer. Since the Cloud layer will archive the data, so the user doesn't need to archive all the information those are generated at the IoT layer. The cloudlet layer will also provide 


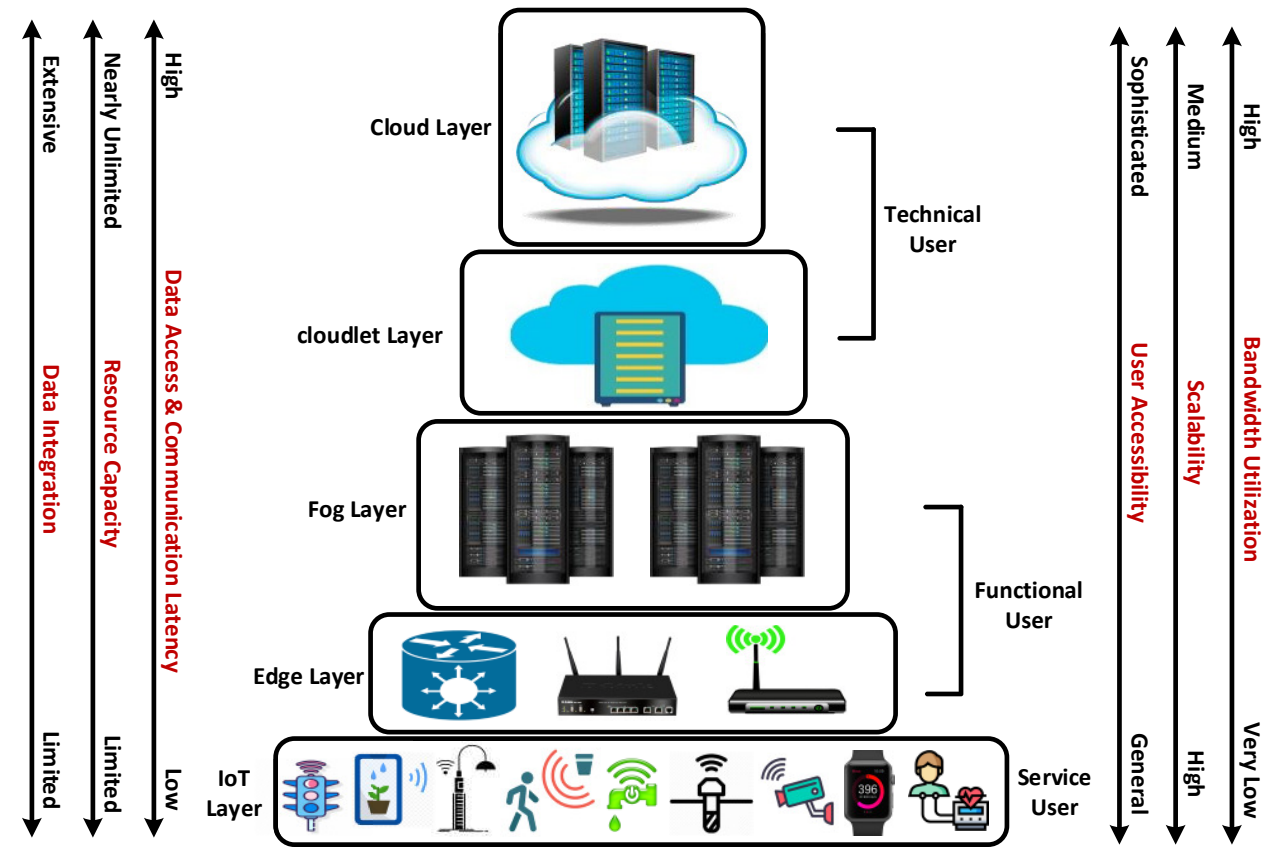

Fig. 3 Layer-wise user accessibility and performance hierarchy of IEF2C framework in a smart city scenario.

detailed information on users and devices such as timing, instance, authorization, location, privacy.

Hence, all the users and devices within a country will be authorized by the cloudlet unit. According to our proposed framework, the cloudlet layer will ensure user and device authentication and mobility features among the smart cities within a country. A user may travel or shift from a city to another within a country. In that case, the user will move from the previous Fog unit to another Fog unit. To be compatible with the new Fog unit, the user and the devices accompanied with will be authorized by the cloudlet layer. Because, both Fog units are under the same cloudlet unit. However, data mobility will not be possible in terms of shifting toward other countries since, the cloudlet combines the cities within a country. When a new user will attempt to login with a unique username and password to an IoT-enabled device of office, hospital, hotel, smart grid, home and any other functional components of a new smart city under an IEF2C framework, cloudlet unit will check the accessibility. At first that IoT-enabled device will send the username and password to the Edge layer. The Edge layer will send the username and password data to the cloudlet layer via the Fog layer. Here, the Fog layer will only relay the data from the Edge to the cloudlet layer. Then, the cloudlet layer will check the authenticity of the user form its database; if it finds the user as an authorized user then it will send authorization to the Edge layer via the Fog layer. Finally, the Edge layer will provide confirmation to the user through the device. Subsequently, if the user accesses the device, the historical data archived in cloud relevant to this device can be accessed. A copy of the authorization database will be replicated periodically to the Cloud.

According to Fig. 3), the cloudlet layer can only be accessed by authorized technical users who will be responsible for data privacy, system upgradation and maintenance.

\subsection{Cloud Layer}

This layer connects all the cloudlet in a common platform of the proposed IEF2C framework. The Cloud stores a huge volume of data simultaneously [17] and is responsible for data privacy according to the business policy. To ensure security, deep computing complex applications will be executed at this layer. Moreover, the layer will provide an interface to the technical users since the Fog unit has a limitation of data storage. In terms of this limitation, the Fog layer transfers its data to the Cloud layer in order to archive. The main objective of the Fog layer is to implement an AI system. For this purpose, it will analyze the data generated at office, hospital, hotel, smart grid, home and any other functional components of a smart city. Eventually, it will send this data to the Cloud and Cloud will archive it. If any disaster (technical and environmental) happens in the Fog layer, the archive will push back to the Fog to recover the system. Moreover, the Cloud aggregates the cloudlets of smart cities. So, data mobility of IoT devices and its a user from one cloudlet to another cloudlet will be possible.

The technical users who are responsible for ensuring proper data management of the cloudlet layer will also maintain the Cloud layer (as shown in Fig. 3). 


\section{Layer-wise Data Types of IEF2C Framework}

According to our proposed IEF2C data management framework of smart cities, each layer of the framework manages the specific type of data (as shown in Fig. 2) such as random data, operational data, authentication data and archive data:

1. Random Data: Random data is generated from the user and IoT devices. The user commands and performance information of devices are defined as random data in the IEF2C data management framework. It will save in the Edge layer for a short time, which is called last recent data [14]. Besides, the Edge layer will have a cache memory. In this layer, all the instructions from the user and the Fog layer will bypass to the IoT layer and viceversa. The random data will be passed to the Fog layer for permanent storing.

2. Operational Data: Operational data is most important in the proposed framework. The Fog layer will also use the operational data to implement artificial intelligence on the IoT layer. The Fog layer will store the data from devices and users then provide to the AI system as required. The Fog layer will need a huge volume of storage capacity to manage the operational data, which will carry maximum information about the city.

3. Authentication Data: Authentication data will be coordinated by the cloudlet layer. The data will carry the user and device information. The information includes general data of user, password, device information, user and device location, data of Edge and Fog units, mobility history of user and device, and other recognized data. Every user and device of the framework will be registered to this layer.

4. Archive Data: Data will be archived in the Cloud layer. It will act as backup and will be restored in the Fog layer at any emergency or disaster period. The Fog layer will send data to the cloudlet layer as well. The cloudlet layer will check and eliminate the unnecessary data and finally send it to the Cloud layer to store it permanently. The Cloud should have a huge volume of data storage to store the archived data. All the data of the framework will be stored in this layer.

\section{IEF2C Framework: Suspected COVID-19 patient detection and contact tracing}

The widespread COVID-19 pandemic situation might be overcome by extensive research on inventing vaccines and medicine

However, as per current statistics it will take at least more 6 months to come to general people. Until then, people have to maintain social distance and other precautionary measures such as wearing masks, cleaning hands frequently. WHO suggests avoiding people gathering, since the virus

\section{Symptoms of COVID-19 affected patients}

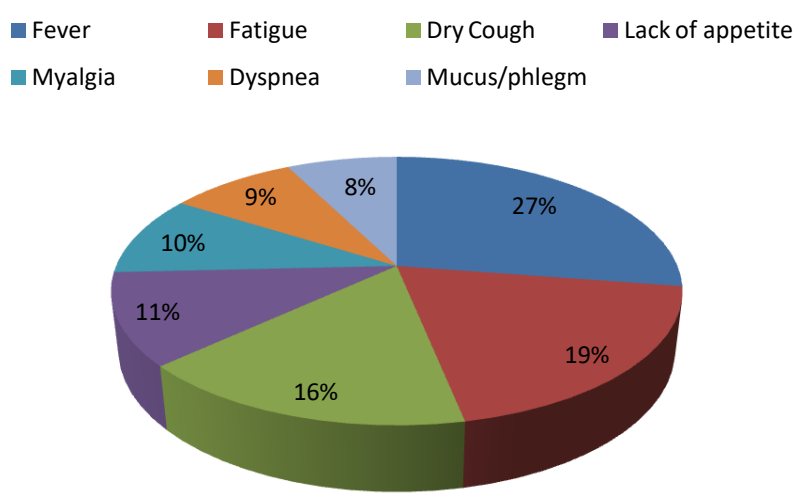

Fig. 4 Symptoms presented with the COVID-19 affected patients.

spreads from man to man spontaneously. The most alarming fact is that the symptoms of COVID-19 are almost similar to other general diseases. Avoiding people gathering is very difficult in the workplace. Therefore, social distancing and isolating the affected people are the most effective ways to prevent this disease.

The researchers have identified the symptoms of COVID19 positive patient, of which few symptoms are commonly exposed for most affected patients and others for seriously sick patients due to chronic diseases. Fig. 4 demonstrates the symptoms presented with the COVID-19 affected [44]. According to the pie chart, it is clear that almost every COVID19 patient suffers from fever. Most of the patients have fatigue and dry cough. Serious patients suffer from dyspnea or shortness of breath.

To mitigate the rate of infection, it is very significant to ensure social distancing and isolation among the people. Identifying the suspected people and ensuring further contact tracing will play a vital role in this regard. In the case of a smart city, suspected COVID-19 patient detection and ensuring contact tracing can be done by using different IoT technologies.

Nowadays, IoT devices can measure instant health information from the users such as the smartwatch can provide body temperature, blood pressure status etc.. Moreover, the pulse oximeter can estimate the breath rate of a user. Smart city data management system will collect different physiological data from wearable IoT devices and analyze to find out the suspected COVID-19 inhabitants of a city and their recent contact. The proposed IEF2C data management framework is exploited for suspected COVID-19 patients detection and ensures contact tracing. Each layer of the proposed IEF2C framework will play important role to find out the suspected patients of a smart city and their social interactions.

In this research, the proposed framework will mark smart COVID-19 suspected people based on two major symptoms - fever and shortness of breath. Those symptoms are consid- 
ered because $99 \%$ of COVID-19 patients suffer from fever and $31 \%$ have shortness of breath [44]. Since, smart city residents use wearable IoT devices, so it is very simple and easy to collect each users body temperature, blood oxygen saturation, pulse rate and blood perfusion index etc.

IoT layer will send the physiological data of every user. Artificial intelligence (AI) system of the Fog unit will analyze those data received from the Edge layer. Different learning patterns (such as supervised learning, unsupervised learning and reinforcement learning) generated by emerging machine learning models (such as kNN, ANN, DNN, CNN and RNN) can be used for observations and experiences with respect to the symptomatic data of COVID-19 [15]. If the information of a user matches with the trained dataset or symptom's parameter then the user will be marked as a suspected COVID-19 patient. Note that, AI system of the Fog layer is not confirmed COVID-19 detection rather it will identify suspected peoples. Such consideration is made as because the users for confirmation of COVID-19 some other health related information need to be tested. So, the user will be marked as suspected not as confirmed case.

\subsection{Function of IoT Sensing Layer}

The IoT layer and the Edge layer of the proposed IEF2C framework will work collaboratively in the IoT sensing layer. IoT sensing layer is introduced for explaining the total data management operation of the proposed data management framework in terms of suspected COVID-19 patient detection and contact tracing.

\subsubsection{Function of IoT Layer}

This layer will communicate with the Edge layer bidirectionally and will play a key role in generating COVID-19 symptomatic data. Body temperature is the input of fever and blood oxygen saturation is the input of shortness of breath; those will be measured using smart wearable IoT devices (such as a smartwatch or any light smart gadgets) and special purpose biosensors. IoT devices and biosensors will send the data of body temperature, blood oxygen saturation, pulse rate and blood perfusion index of a user. Moreover, the Edge layer will provide the suspected user information to the applications of the user devices. Those user applications can be mobile application or home monitoring software. Suspected users and their close contacted people at the same Edge unit will be alert from the upper layer through the user applications.

\subsubsection{Function of Edge Layer}

The Edge layer will share the data as a hub among the users and the Fog layer. When the symptomatic data of a user gen- erate in the user's IoT devices, the Edge will collect the data and deliver it to the Fog layer for AI-based analysis. After completing the analysis, the Fog layer will send the result to the Edge unit. Then the Edge unit will share the health alert to the COVID-19 suspected user and other surrounding users to make aware of the suspected COVID-19 patient at the same Edge unit.

The Edge layer will also act as a locator. The layer will be exploited to inform the location of the suspected COVID19 patient to the surrounding people under a common Edge unit. Movement of the suspected COVID-19 patients from one Edge to another can also be detected because the Edge units will be clustered under a Fog unit. Thus, the contact tracing process will be ensured.

\subsection{Function of Fog Layer}

The Fog layer will play the main role in this regard. It will collect user health data generated at IoT devices. Then the AI system models (such as kNN, ANN, DNN, CNN and RNN) will analyze the data with respect to the user's previous health information and current COVID-19 symptoms parameters such as body temperature, blood oxygen saturation, pulse rate and blood perfusion index. If the health information matches the trained pattern parameters then the user will be marked as COVID-19 suspected. So, other people being notified will be alerted in this regard. Based on the symptomatic data of three consecutive days in a Fog database, the suspected users will be assigned with different status.

To analyze the user's health condition, the AI system of the Fog layer will use the user's consecutive three days' health data as well as symptomatic data. Then the machine learning algorithms of models at Fog layer will analyze all the health data and provide a possible prediction. Eventually, this layer will distribute the predicted result to the cloudlet layer and IoT sensing layer via Edge unit.

The statistics of the suspected people will help to monitor the pandemic situation in a city and city authority will be able to take the proper steps. The isolation process will be easy by using the prediction of COVID-19 suspected detection. It will also help to stop the movement of suspects. In this regard, the location of the suspected users will be monitored through the Fog layer. A summary of the suspected users will be sent to the cloudlet layer and the central health monitoring organization of a country.

\subsection{Function of cloudlet Layer}

In this research, a cloudlet unit will be represented as a hub of all the cities of a country that collect the information from the Fog unit of each city. When a user moves from one city 


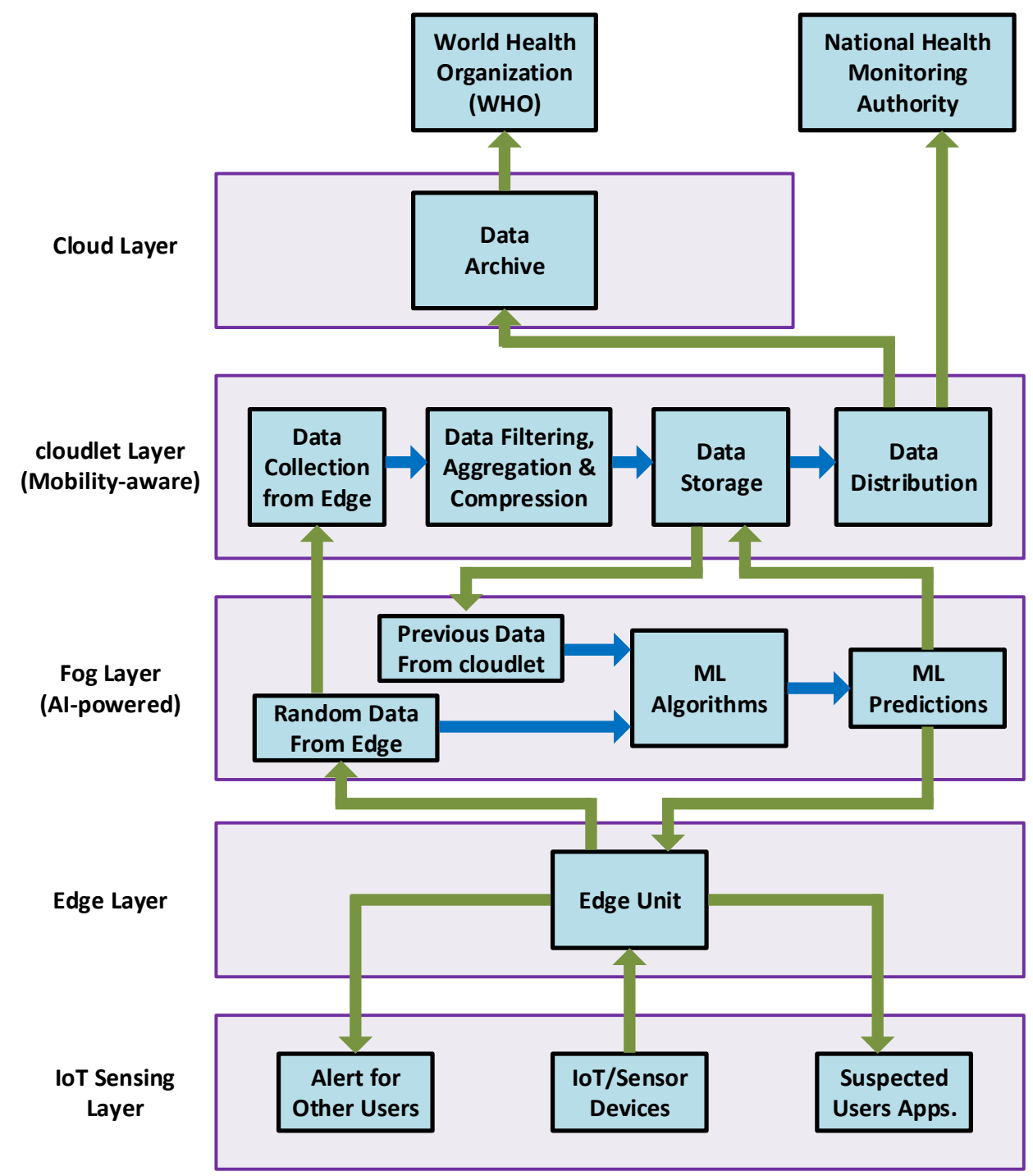

Fig. 5 Suspected COVID-19 patient detection and contact tracing based on IEF2C framework.

to another city the cloudlet layer will confirm the authentication and will provide access to smart city features in the new city. The cloudlet layer will store the user's health data and provide the national health monitoring authority. The cloudlet layer will store the user's all types of data (previous and recent data), and distribute the data to the Fog and Cloud layer as per demand.

The cloudlet layer will also share the suspected user's information with the national health monitoring authority. The concerned authority will get access to know the proper information about the suspected users. So, it will be easy to take the proper step about the suspected user and the surrounding people for contact tracing. Periodically, the cloudlet layer will send the information of COVID-19 to the cloud layer. So, the data will have a permanent backup and multi-sources. Authority can easily reach to the suspected person for test- ing COVID-19 and as a result the government will be able to take the proper steps in this regard.

Staying in a certain place and not moving to another place is one of the most important conditions to mitigate pandemic situation. If the movement of the inhabitants from one city to another is stopped, then the rate of affected people will decrease significantly. It will be possible by using the cloudlet layer. When a user logs out from a Fog unit and try to $\log$ in to another Fog unit, the cloudlet layer will detect it and will inform the proper authority in this regard.

\subsection{Function of Cloud Layer}

The COVID-19 related data of the user will be archived in Cloud. Eventually, it will help to understand the total scenario of the countrywide pandemic. The World Health Organization (WHO) is not able to provide guidelines due to 
lack of sufficient information. In this regard, the cloud data will WHO to provide proper guidelines. Moreover, it will help to understand the characteristics and future perceptions situations about the pandemic.

\section{Conclusion}

Smart city technology is an emerging research issue and cutting-edge technology of modern civilization where citizens as an essential part connected to a network. To coordinate all the components of smart city properly, an efficient and effective data management system is needed. This research proposes efficient data management framework termed as IEF2C, which will perform in different computing layers. Due to the division of the whole architecture in layers, the data traffic load and latency will be decreased; likewise, synchronized management will be developed. Fog layer will be exploited as a cluster so that it becomes more effective in developing the AI system in minimum time. The Edge layer will coordinate the IoT layer and create the interconnectivity among the ends. Finally, the proposed AI-powered IEF2C data management framework is exploited for suspected COVID-19 patient detection and contact tracing in smart cities. Till now, the COVID-19 testing facility is not sufficient with respect to demand and the number of affected COVID-19 patients is increasing day by day. The proposed IEF2C framework will analyze the citizens' symptomatic health data using machine learning algorithm and alert the COVID-19 suspected citizens without testing. In the future, we will compare our proposed contact tracing strategy with state-of-the-art strategies to perform the performance analysis of the proposed framework.

\section{Conflict of interest}

The authors declare that they have no conflict of interest.

\section{References}

1. D. Celentano, J. Szklo, and M. Szklo, Gordis Epidemiology. Elsevier, 2018. [Online]. Available: https://books.google.com.bd/ books?id=Xwl0DwAAQBAJ

2. H. N. Joann Elmore, Dorothea Wild and D. Katz, Jekel's Epidemiology, Biostatistics, Preventive Medicine, and Public Health. Elsevier, 2020. [Online]. Available: https://www.elsevier.com/books/ jekels-epidemiology-biostatistics-preventive-medicine-and-public-health/ elmore/978-0-323-64201-9

3. W. H. Organization, "Coronavirus disease 2019 (covid-19): situation report, 51," Technical documents, 2020-03-11. [Online]. Available: https://www.who.int/docs/default-source/ coronaviruse/situation-reports/20200311-sitrep-51-covid-19. pdf?sfvrsn=1ba62e57_10
4. World Health Organization, "Emerging respiratory viruses, including covid-19: methods for detection, prevention, response and control," https://openwho.org/courses/introduction-to-ncov, accessed: 2020-08-22.

5. L. Fu, B. Wang, T. Yuan, X. Chen, Y. Ao, T. Fitzpatrick, P. Li, Y. Zhou, Y. fan Lin, Q. Duan, G. Luo, S. Fan, Y. Lu, A. Feng, Y. Zhan, B. Liang, W. Cai, L. Zhang, X. Du, L. Li, Y. Shu, and H. Zou, "Clinical characteristics of coronavirus disease 2019 (covid-19) in china: A systematic review and meta-analysis," Journal of Infection, vol. 80, no. 6, pp. 656 - 665, 2020. [Online]. Available: http: //www.sciencedirect.com/science/article/pii/S0163445320301705

6. S. Poudel, S. Meng, Y.-J. Wu, Y.-P. Mao, R.-X. Ye, Q.-Z. Wang, C. Sun, S. Sylvia, S. Rozelle, H. Raat, and H. Zhou, "Epidemiology, causes, clinical manifestation and diagnosis, prevention and control of coronavirus disease (covid-19) during the early outbreak period: A scoping review,' Infectious Diseases of Poverty, vol. 9, 03 2020. [Online]. Available: http://dx.doi.org/10.1186/s40249-020-00646-x

7. R. P. Kotian, D. Faujdar, S. P. Kotian, and B. D'souza, "Knowledge and understanding among medical imaging professionals in india during the rapid rise of the covid-19 pandemic," Health and Technology, may 2020. [Online]. Available: https://doi.org/10. 1007/s 12553-020-00437-2

8. W. H. Organization, "Coronavirus disease 2019 (covid-19): situation report, 116," Technical documents, 2020-05-15. [Online]. Available: https://www.who.int/docs/default-source/ coronaviruse/situation-reports/20200515-covid-19-sitrep-116. pdf?sfvrsn=8dd60956_2

9. A. Hussain, B. Bhowmik, and N. Moreira, "Covid-19 and diabetes: Knowledge in progress," Diabetes Research and Clinical Practice, vol. 162, p. 108142, 042020.

10. "Centre for disease control and prevention, coronavirus disease 2019 (covid-19)." https://www.cdc.gov/coronavirus/2019-ncov/ symptoms-testing/symptoms.html, accessed: 2020-08-23.

11. C. Huang, Y. Wang, X. Li, L. Ren, J. Zhao, Y. Hu, L. Zhang, G. Fan, J. Xu, X. Gu, Z. Cheng, T. Yu, J. Xia, Y. Wei, W. Wu, X. Xie, W. Yin, H. Li, M. Liu, Y. Xiao, H. Gao, L. Guo, J. Xie, G. Wang, R. Jiang, Z. Gao, Q. Jin, J. Wang, and B. Cao, "Clinical features of patients infected with 2019 novel coronavirus in wuhan, china," The Lancet, vol. 395, no. 10223, pp. 497 - 506, 2020. [Online]. Available: http: //www.sciencedirect.com/science/article/pii/S0140673620301835

12. W. H. Organization, "Contact tracing in the context of covid-19: interim guidance, 10 may 2020," Technical documents, 2020.

13. J. Jin, J. Gubbi, S. Marusic, and M. Palaniswami, "An information framework for creating a smart city through internet of things," IEEE Internet of Things Journal, vol. 1, pp. 112-121, 2014.

14. A. Sinaeepourfard, J. Krogstie, S. A. Petersen, and D. Ahlers, "F2c2c-dm: A fog-to-cloudlet-to-cloud data management architecture in smart city," in 2019 IEEE 5th World Forum on Internet of Things (WF-IoT), 2019, pp. 590-595.

15. F. Samie, L. Bauer, and J. Henkel, "From cloud down to things: An overview of machine learning in internet of things," IEEE Internet of Things Journal, vol. 6, no. 3, pp. 4921-4934, June 2019.

16. R. Rivera, J. G. Robledo, V. M. Larios, and J. M. Avalos, "How digital identity on blockchain can contribute in a smart city environment,' in 2017 International Smart Cities Conference (ISC2), Sep. 2017, pp. 1-4.

/ I. Sitton-Candanedo, R. Alonso, O. Garcia, L. Munoz, and S. Rodriguez, "Edge computing, iot and social computing in smart energy scenarios," Sensors, vol. 19, p. 3353, 072019.

18. M. Rahman, R. Khan, M. Khandaker, and M. S. Ar Salan, "An automated contact tracing approach for controlling covid-19 spread based on geolocation data from mobile cellular networks," 07 2020 . 
19. J. Bay, J. Kek, A. Tan, and C. S. Hau, "Bluetrace: A privacypreserving protocol for community-driven contact tracing across borders," 2020.

20. R. Sonkin, E. A. Alpert, and E. Jaffe, "Epidemic investigations within an arm's reach - role of google maps during an epidemic outbreak," Health and Technology, 072020.

21. K. A. Achmad, L. E. Nugroho, A. Djunaedi, and Widyawan, "Smart city model: a literature review," in 2018 10th International Conference on Information Technology and Electrical Engineering (ICITEE), July 2018, pp. 488-493.

22. C. A. Ramirez, R. C. Barragan, G. Garcia-Torales, and V. M. Larios, "Low-power device for wireless sensor network for smart cities," in 2016 IEEE MTT-S Latin America Microwave Conference (LAMC), Dec 2016, pp. 1-3.

23. N. Villanueva-Rosales, Ruey Long Cheu, A. Gates, N. Rivera, O. Mondragon, S. Cabrera, C. Ferregut, C. Carrasco, S. Nazarian, H. Taboada, V. M. Larios, L. Barbosa-Santillan, M. Svitek, O. Pribyl, T. Horak, and D. Prochazkova, "A collaborative, interdisciplinary initiative for a smart cities innovation network," in 2015 IEEE First International Smart Cities Conference (ISC2), Oct 2015, pp. 1-2.

24. A. Arman, A. Abbas, and R. Hurriyati, "Analysis of smart city technology initiatives for city manager to improve city services and quality of life based on iso 37120," 112015.

25. "Edge intelligence makes smart homes truly intelligent." https://connect.altran.com/2019/06/ edge-intelligence-makes-smart-homes-truly-intelligent/, accessed: 2020-08-23.

26. A. Sinaeepourfard, J. Garcia, X. Masip-Bruin, E. Marin-Tordera, $\mathrm{X}$. Yin, and C. Wang, "A data lifecycle model for smart cities," in 2016 International Conference on Information and Communication Technology Convergence (ICTC), Oct 2016, pp. 400-405.

27. A. Sinaeepourfard, J. Garcia, X. Masip-Bruin, and E. MarinTordera, "A novel architecture for efficient fog to cloud data management in smart cities," in 2017 IEEE 37th International Conference on Distributed Computing Systems (ICDCS), June 2017, pp. $2622-2623$.

28. A. Sinaeepourfard, J. Krogstie, S. A. Petersen, and A. Gustavsen, "A zero emission neighbourhoods data management architecture for smart city scenarios: Discussions toward 6vs challenges," in 2018 International Conference on Information and Communication Technology Convergence (ICTC), Oct 2018, pp. 658-663.

29. T. N. Gia, M. Jiang, A. Rahmani, T. Westerlund, P. Liljeberg, and H. Tenhunen, "Fog computing in healthcare internet of things: A case study on ecg feature extraction," in 2015 IEEE International Conference on Computer and Information Technology; Ubiquitous Computing and Communications; Dependable, Autonomic and Secure Computing; Pervasive Intelligence and Computing, Oct 2015, pp. 356-363.

30. H. Moura, C. Andre da Costa, R. Righi, and R. Antunes, "Fog computing in health: A systematic literature review," Health and Technology, 052020.

31. B. Farahani, F. Firouzi, V. I. Chang, M. Badaroglu, N. Constant, and K. Mankodiya, "Towards fog-driven iot ehealth: Promises and challenges of iot in medicine and healthcare," Future Gener. Comput. Syst., vol. 78, pp. 659-676, 2018.

32. J. Colistra, "The evolving architecture of smart cities," in 2018 IEEE International Smart Cities Conference (ISC2), Sep. 2018, pp. 1-8.

33. A. M. Rahmani, T. Nguyen gia, B. S. Negash, A. Anzanpour, I. Azimi, M. Jiang, and P. Liljeberg, "Exploiting smart e-health gateways at the edge of healthcare internet-of-things: A fog computing approach," Future Generation Computer Systems, 022017.

34. Q. Zhu, R. Wang, Q. Chen, Y. Liu, and W. Qin, "Iot gateway: Bridgingwireless sensor networks into internet of things," in 2010 IEEE/IFIP International Conference on Embedded and Ubiquitous Computing, Dec 2010, pp. 347-352.
35. I. Azimi, A. Anzanpour, A. M. Rahmani, T. Pahikkala, M. Levorato, P. Liljeberg, and N. Dutt, "Hich: Hierarchical fog-assisted computing architecture for healthcare iot," $A C M$ Trans. Embed. Comput. Syst., vol. 16, no. 5s, Sep. 2017. [Online]. Available: https://doi.org/10.1145/3126501

36. M. Bhatia and S. K. Sood, "Exploring temporal analytics in fog-cloud architecture for smart office healthcare," Mob. Netw. Appl., vol. 24, no. 4, p. 1392 to 1410, Aug. 2019. [Online]. Available: https://doi.org/10.1007/s11036-018-0991-5

37. K. Halunen and V. Ollikainen, "Rendezvous based pandemic tracing by sharing diffie-hellman generated common secrets," 04 2020.

38. M. Whaiduzzaman, M. R. Hossain, A. R. Shovon, S. Roy, A. Laszka, R. Buyya, and A. Barros, "A privacy-preserving mobile and fog computing framework to trace and prevent covid-19 community transmission," ArXiv, vol. abs/2006.13364, 2020.

39. E. Hernandez-Orallo, P. Manzoni, C. T. Calafate, and J. Cano, "Evaluating how smartphone contact tracing technology can reduce the spread of infectious diseases: The case of covid-19," IEEE Access, vol. 8, pp. 99 083-99 097, 2020.

40. N. Ahmed, R. A. Michelin, W. Xue, S. Ruj, R. Malaney, S. S. Kanhere, A. Seneviratne, W. Hu, H. Janicke, and S. K. Jha, "A survey of covid-19 contact tracing apps," IEEE Access, vol. 8, pp. 134 577-134 601, 2020.

41. T. K. Burki, "Testing for covid-19," The Lancet Respiratory Medicine, 2020. [Online]. Available: https://doi.org/10.1016/ S2213-2600(20)30247-2

42. W. Zhao, Z. Zhong, X. Xie, Q. Yu, and J. Liu, "Relation between chest ct findings and clinical conditions of coronavirus disease (covid-19) pneumonia: A multicenter study," American Journal of Roentgenology, vol. 214, pp. 1-6, 032020.

43. Y. Li and L. Xia, "Coronavirus disease 2019 (covid-19): Role of chest ct in diagnosis and management," American Journal of Roentgenology, vol. 214, pp. 1-7, 032020.

44. "Coronavirus and covid-19: What you should know." https://www. webmd.com/lung/coronavirus, accessed: 2020-08-23. 
Figures

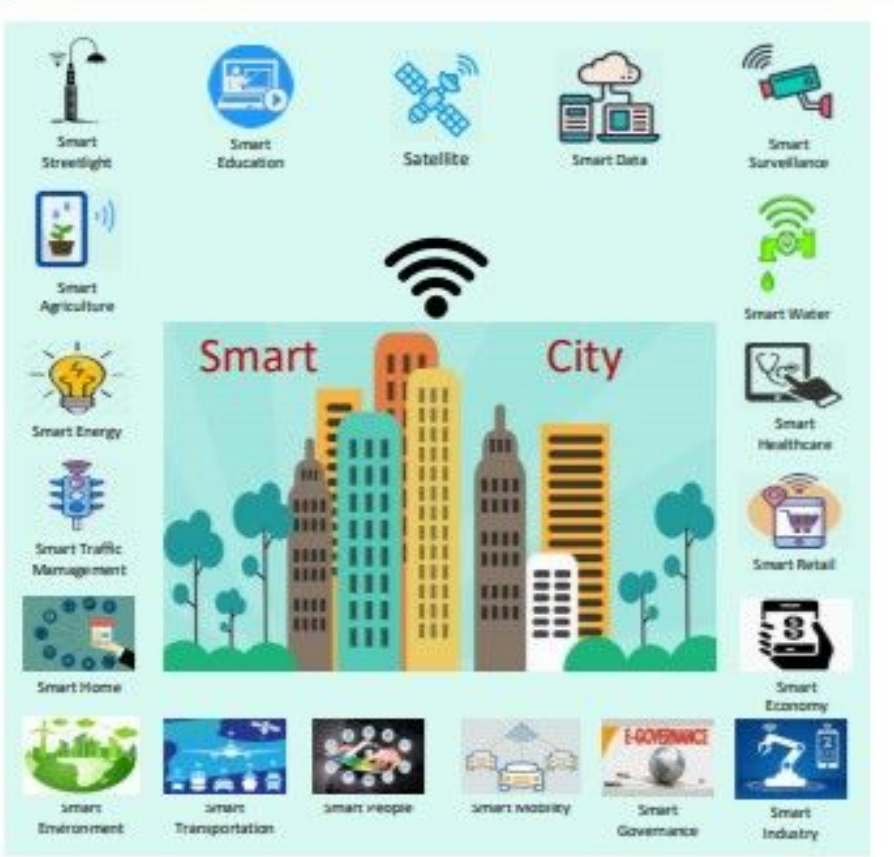

Figure 1

Smart city architecture overview 


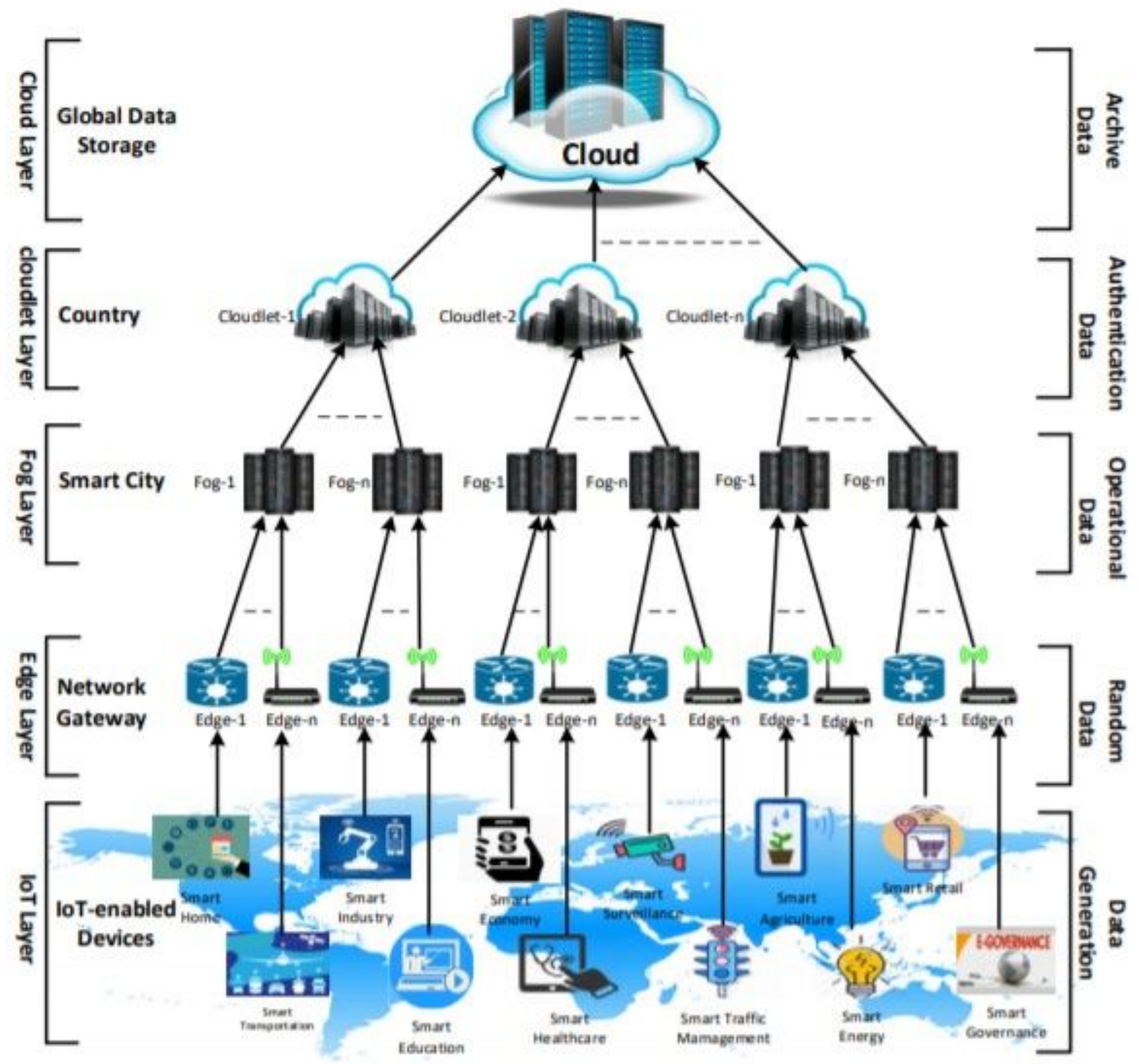

Figure 2

IEF2C data management framework of a smart city scenario 


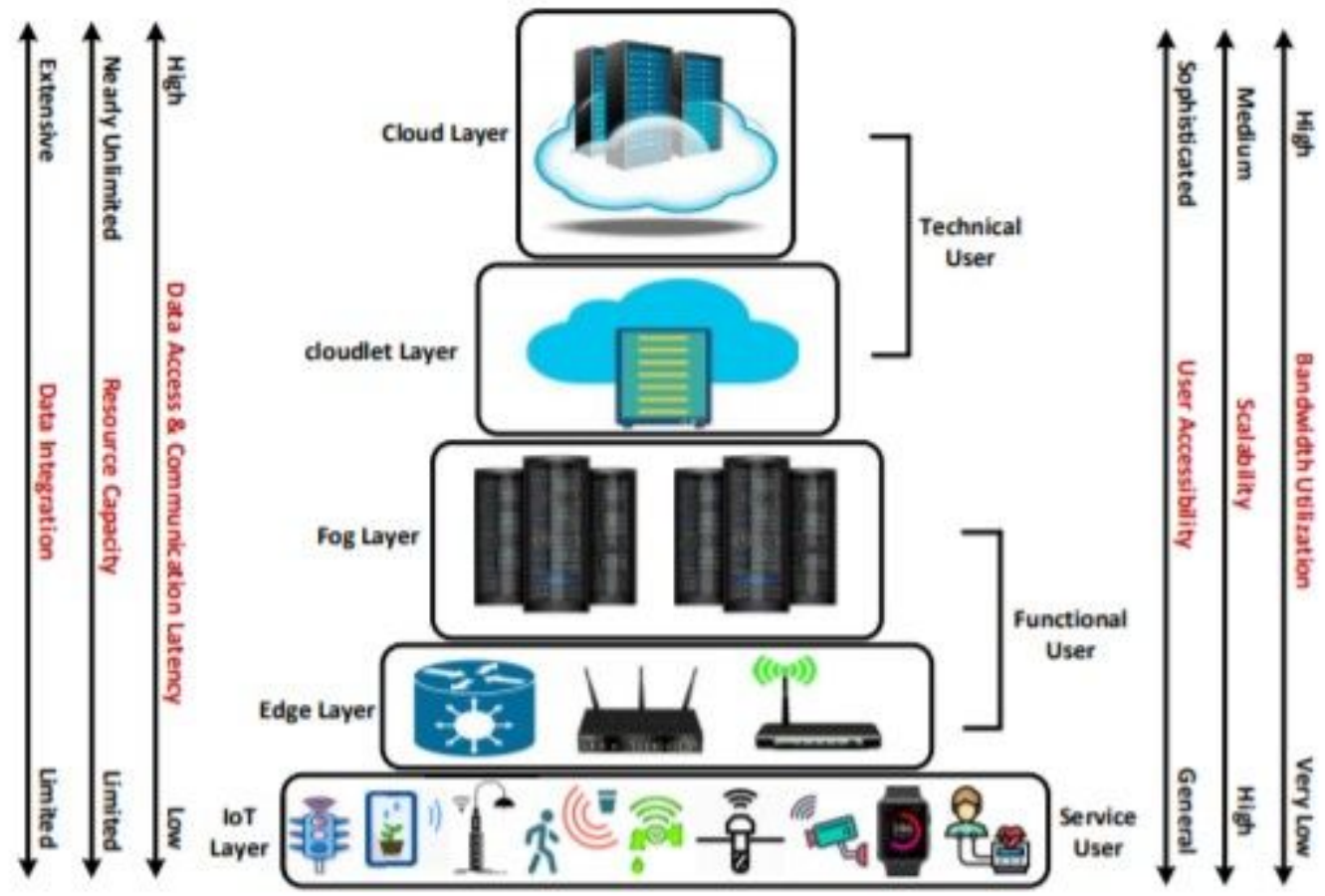

Figure 3

Layer-wise user accessibility and performance hierarchy of IEF2C framework in a smart city scenario

Symptoms of COVID-19 affected patients

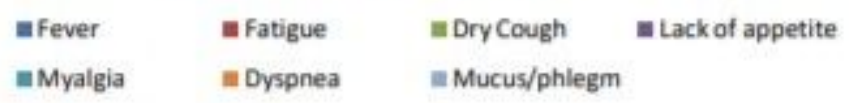

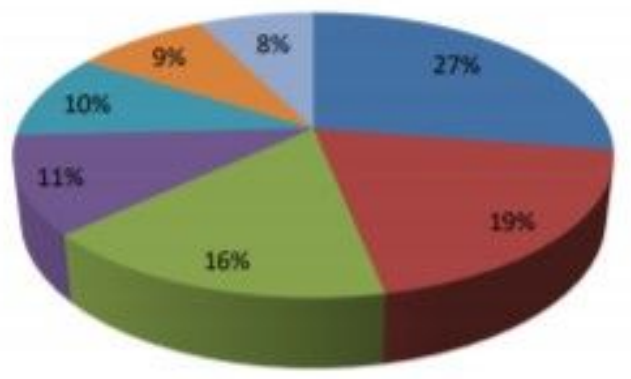

Figure 4

Symptoms presented with the COVID-19 affected patients 


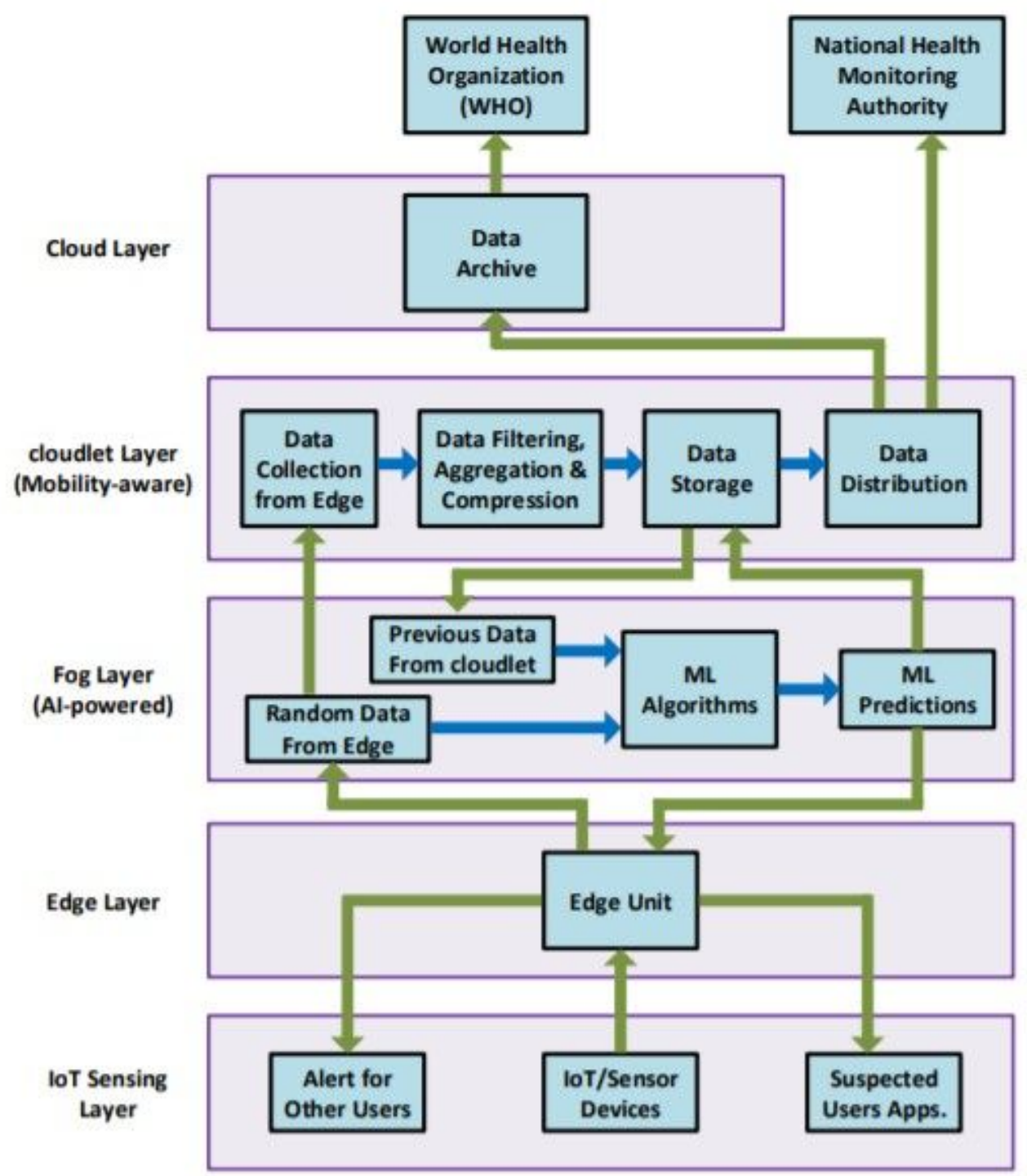

Figure 5

Suspected COVID-19 patient detection and contact tracing based on IEF2C framework 